\title{
REPRESENTING TRIPLES OF A SYMPLECTIC MANIFOLD
}

\author{
GUIDO KARRER
}

ABSTRACT. For a symplectic manifold $M$ and its associated real Lie algebra $P(M, \omega)$ (its Poisson algebra) a definition of first-order representations and a structure theorem for the representation ring is given.

0. In physics and in the context of geometric quantization, the term prequantization is used to denote a special kind of representation of the Poisson algebra $P(M, \omega)$. Here, we give a more general and natural definition of representations of the Lie algebra $P(M, \omega)$ (Definition 1) and show, how prequantizations fit into this scheme, and we finally compute the representation ring (Propositions 4, 5). We rely on some facts on prequantizations, which can be found in $[1,2,3]$; as for differential operators, we refer to [4].

1. In the sequel we need the following statements on prequantization. Let $(M, \omega)$ denote a symplectic manifold, i.e. a paracompact, connected, real $C^{\infty}$-manifold of dimension $n$ carrying a regular and closed 2 -form $\omega$; let $C^{\infty}(M)$ denote the ring of real valued $C^{\infty}$-functions on $M$. Every $f \in C^{\infty}(M)$ has its Hamilton field $v_{f} \in$ $C^{\infty}(M)$ given by $d f=\omega\left(-, v_{f}\right)$; then, the Poisson brackets \{\}$: C^{\infty}(M) \times C^{\infty}(M) \rightarrow$ $C^{\infty}(M)$ are defined by

$$
\{f, g\}:=\omega\left(v_{f}, v_{g}\right)=v_{f} \cdot g \text { for } f, g \in C^{\infty}(M) .
$$

The real Lie algebra $\left(C^{\infty}(M),\{\}\right)$ is the Poisson algebra of $(M, \omega)$ and denoted by $P(M, \omega)$.

On the other hand, let $\lambda$ be a complex line bundle over $M$ with bundle space $E$ and section module $\Gamma(\lambda)$, let $\alpha \in \mathbf{C}$, and let $\operatorname{Diff}(\lambda)$ be the $\mathbf{C}$-vector space of $\mathbf{C}$-linear local operators on $\Gamma(\lambda)$. An $\alpha$-prequantization of $(M, \omega)$ in the bundle $\lambda$ is a Lie algebra homomorphism $D: P(M, \omega) \rightarrow \operatorname{Diff}(\lambda)$ of the form

$$
D f=\nabla\left(v_{f}\right)+\alpha f, \quad f \in C^{\infty}(M),
$$

$\nabla$ a linear connection on $\lambda, \nabla\left(v_{f}\right)$ its covariant derivative in direction of $v_{f}$. Note that $\nabla$ and $\alpha$ are determined by $D$.

If $\kappa(\nabla)$ denotes the curvature form of $\nabla,(1.1)$ implies

$$
D\{f, g\}=D f \circ D g-D g \circ D f \quad \forall f, g \in C^{\infty}(M) \Leftrightarrow \kappa(\nabla)=-\alpha \omega .
$$

The first Chern class $c_{1}(\lambda)=(1 / 2 \pi i)[\kappa(\nabla)] \in H^{2}(M, \mathbf{C})$ is integral, i.e. $c_{1}(\lambda) \in$ $\iota_{*} H^{2}(M, \mathbf{Z})$, where $\iota_{*}: H^{2}(M, \mathbf{Z}) \rightarrow H^{2}(M, \mathbf{C})$ is induced by $\iota: \mathbf{Z} \rightarrow \mathbf{C}$.

For $(M, \omega)$ given, we say that $\alpha \in \mathbf{C}$ is a quantum number of $(M, \omega)$ if $(\alpha / 2 \pi i)[\omega] \in$ $H^{2}(M, \mathbf{C})$ is integral; the Z-module $Q(M, \omega)$ of quantum numbers of $(M, \omega)$ satisfies

$$
\begin{aligned}
Q(M, \omega) & =\mathbf{C} \quad \text { if }[\omega]=0 \\
\operatorname{dim}_{\mathbf{Z}} Q(M, \omega) & =0 \text { or } 1 \quad \text { if }[\omega] \neq 0 \quad[2, \text { II Satz 6.3]. }
\end{aligned}
$$

Received by the editors December 2, 1980.

1980 Mathematics Subject Classification. Primary 17B65, 22E65; Secondary 81C99. 
A prequantization $(D, \lambda)$ with associated linear connection $\nabla$ is metrizable means that there exists a positive-definite Hermitian form $H$ on $\lambda$ with $\nabla H=0$. (1.2) implies that the associated quantum number $\alpha$ of $(D, \lambda)$ is purely imaginary.

PROPOSITION 1. To every (purely imaginary) quantum number $\alpha$ of $(M, \omega)$ there exists a (metrizable) $\alpha$-prequantization $(D, \lambda)$ of $(M, \omega)$.

An isomorphism $\phi:(D, \lambda) \rightarrow\left(D^{\prime}, \lambda^{\prime}\right)$ of prequantizations of $(M, \omega)$ is a bundle isomorphism $\lambda \rightarrow \lambda^{\prime}$ commuting with the operations of $f \in C^{\infty}(M)$ on $\lambda$ and $\lambda^{\prime}$ respectively.

Proposition 2. Let $\iota_{1 *}: H^{1}(M, \mathbf{Z}) \rightarrow H^{1}(M, \mathbf{C})\left(\iota_{2 *}: H^{\prime}(M, \mathbf{Z}) \rightarrow H^{\prime}(M, \mathbf{R})\right)$ be induced by $\iota_{1}: \mathbf{Z} \rightarrow \mathbf{C}\left(\iota_{2}: \mathbf{Z} \rightarrow \mathbf{R}\right)$, and let $\alpha$ be a fixed (purely imaginary) quantum number of $(M, \omega)$. Then, the set of isomorphism classes of (metrizable) $\alpha$-prequantizations of $(M, \omega)$ is isomorphic to $\operatorname{coker} \iota_{1 *}$ (coker $\iota_{2 *}$ resp.), in particular $\cong$ pt $\}$ if $H_{1}(M, \mathbf{Z})=0 ;$ and $\cong H^{1}\left(M, \mathbf{C}^{*}\right)\left(\cong H^{1}\left(M, S^{1}\right)\right.$ resp. $)$ if $H_{1}(M, \mathbf{Z})$ is free.

2. We generalize now the concept of prequantization by Definition 1 .

DEFINITION 1. A first-order representation of the symplectic manifold $(M, \omega)$ is a pair $(D, \xi)$ of a complex vector bundle $\xi$ over $M$ and a real Lie algebra homomorphism $D: P(M, \omega) \rightarrow \operatorname{Diff}(\xi)$ such that

$$
D \text { is local, } \operatorname{ord}(D) \leq 1 \text {; }
$$

$$
(D f)(g s)=\{f, g\} s+g((D f) s) \quad \forall f, g \in C^{\infty}(M), s \in \Gamma(\xi) .
$$

Let us recall some definitions of terms used above: if $\xi$ is a complex vector bundle over $M, \psi$ a local $\mathbf{C}$-linear operator $\Gamma(\xi) \rightarrow \Gamma(\xi)$ and $x \in M, \operatorname{ord}(\psi, x) \leq n \in \mathbf{N}$ means

$$
\psi\left(\left(\prod_{i=1}^{n+1} f_{i}\right) s\right)(x)=0 \quad \forall f_{i} \in C^{\infty}(M, \mathbf{C}), f_{i}(x)=0, s \in \Gamma(\xi) .
$$

ord $\psi \leq n \in \mathbf{N}$ means $\operatorname{ord}(\psi, x) \leq n \forall x \in M$, and if ord $\psi \leq n$, the symbol $\Sigma_{\psi}$ of $\psi$ is defined as the symmetric bundle map of $n$ arguments

$$
\Sigma_{\psi}: \tau^{*}(M) \otimes \cdots \otimes \tau^{*}(M) \rightarrow \text { End } \xi,
$$

which, at a point $x \in M$, is given by

$$
\Sigma_{\psi}\left(d f_{1}(x), \ldots, d f_{n}(x)\right) s(x):=\left(\left(\prod_{i=1}^{n} f_{i}\right) s\right)(x) \forall f_{i} \in C^{\infty}(M), s \in \Gamma(\xi), f_{i}(x)=0 .
$$

DEFINITION 2. A representing triple of $(M, \omega)$ is a triple $(\xi, \nabla, \psi)$ of a complex vector bundle $\xi$ over $M$, a linear connection $\nabla$ on $\xi$, and a bundle map $\psi: \xi \rightarrow \xi$ such that

$$
\begin{aligned}
\nabla \psi & =0 ; \\
K(\nabla) & =-\omega \psi ;
\end{aligned}
$$

where $K(\nabla) \in \Omega^{2}(M, \mathbf{C}) \otimes$ End $\xi$ is the curvature of $\nabla$.

PROPOSITION 3. The set of first-order representations $(D, \xi)$ is isomorphic to the set of representing triples $(\xi, \nabla, \psi)$ of $(M, \omega)$, the isomorphism being given by

$$
D f=\nabla\left(v_{f}\right)+f \psi \quad \forall f \in C^{\infty}(M) .
$$


Proof. Take a first-order representation $(D, \xi)$ of $(M, \omega)$. (2.2) implies that, if $f \in C^{\infty}(M), D f$ is a linear differential operator $\xi \rightarrow \xi$ with ord $D f \leq 1$. Locally $D f$ can be expressed by means of an arbitrary linear connection $\stackrel{\circ}{\nabla}$ on $\xi$ :

$$
(D f) s=\sum_{i=1}^{n} \sigma^{i}(f) \stackrel{\circ}{\nabla}\left(v_{i}\right) s+\psi(f) s
$$

where $v_{i}, i=1, \ldots, n$, is a local vector field-basis of $M, \sigma^{i}(f) \in$ End $\xi$ and $\psi(f) \in$ End $\xi$ are local bundle maps. Again from (2.2) follows

$$
\sum_{i=1}^{n} \sigma^{i}(f)\left(v_{i} \cdot g\right)=\{f, g\} \mathbf{1}=\left(v_{f} \cdot g\right) \mathbf{1}, \quad \forall g \in C^{\infty}(M, \mathbf{C}), \mathbf{1}=\mathrm{id}: \xi \rightarrow \xi
$$

As the two differential operators $\sum_{i=1}^{n} \sigma^{i}(f) \stackrel{\circ}{\nabla}\left(v_{i}\right)$ and ${ }^{\circ}\left(v_{f}\right)$ have the same symbol, they differ by an endomorphism of $\xi$, thus $D f$ has the form

$$
D f=\stackrel{\circ}{\nabla}\left(v_{f}\right)+\stackrel{\circ}{\psi}(f) \text {. }
$$

Now, condition (2.1) implies that $\dot{\psi}$ is a linear differential operator $M \times \mathbf{R} \rightarrow$ End $\xi$ of order $\leq 1$. Let $\alpha: \tau^{*}(M) \rightarrow$ End $\xi$ be its symbol; then, $C^{\infty}(M) \mid \ni f \mapsto \alpha(d f)$ is again a linear differential operator $M \times \mathbf{R} \rightarrow \operatorname{End} \xi$, so $\stackrel{\circ}{\psi}(f)-\alpha(d f)=f \psi$ for some $\psi \in$ End $\xi$. Under the isomorphism $\tau^{*}(M) \rightarrow \tau(M)$ induced by $\omega, \alpha(d f)=\beta\left(v_{f}\right)$ for a bundle map $\beta: \tau(M) \rightarrow$ End $\xi$, and the linear connection $\nabla:=\nabla^{\circ}+\beta$ finally satisfies $D f=\nabla\left(v_{f}\right)+f \psi$. The fact that $D$ preserves the Poisson brackets is equivalent to the statement

$$
0=K(\nabla)\left(v_{f}, v_{g}\right)+\{f, g\} \psi+\left(g \nabla\left(v_{f}\right)-f \nabla\left(v_{g}\right)\right) \psi
$$

which implies (for $g=1$ ):

$$
0=\nabla\left(v_{f}\right) \psi \quad \forall f \in C^{\infty}(M),
$$

hence $\nabla \psi=0$, and $\omega\left(v_{f}, v_{g}\right)=\{f, g\}$ transforms (2.7) into (2.4).

3. Suppose a representing triple $(\xi, \nabla, \psi)$ of $(M, \omega)$ is metrizable, i.e. there exists a positive-definite Hermitian form $H$ on $\xi$ such that $\nabla H=0$. Then, at each point $x \in M$ and for $v_{x}, w_{x} \in T_{x}(M), K(\nabla)\left(v_{x}, w_{x}\right)$ is a skew-endomorphism $E_{x} \rightarrow E_{x}$ in the fiber of $\xi$ with respect to $H$ and as such decomposes into the eigenspaces corresponding to purely imaginary eigenvalues $\alpha_{i} E_{x}:=E\left(\alpha_{1}\right) \oplus \cdots \oplus E\left(\alpha_{r}\right)$. So, in a metrizable triple $(\xi, \nabla, \psi), K(\nabla)=-\omega \psi$ and the fact that $\omega$ is a real 2-form, imply the complete reducibility of $\psi: \xi \rightarrow \xi$ at each point $x \in M$ corresponding to purely imaginary eigenvalues of $\psi_{x}$. Then $\nabla \psi=0$ and the connectedness of $M$ yield

LEMMA 1. A metrizable representing triple $(\xi, \nabla, \psi)$ of $(M, \omega)$ has unique subbundles $\xi_{1}, \ldots, \xi_{r}$ of $\xi$ and purely imaginary numbers $\alpha_{1}, \ldots, \alpha_{r}$, such that $\xi=$ $\bigoplus_{i=1}^{r} \xi_{r}, \xi_{i}$ is the eigenbundle to the eigenvalue $\alpha_{i}$ of $\psi$ invariant under $\nabla$ and with induced connection $\nabla_{i}$, hence $\nabla=\bigoplus_{i=1}^{r} \nabla_{i}$.

COROLlARY. A metrizable first-order representation $(D, \xi)$ of $(M, \omega)$ is a unique direct sum of metrizable first-order representations $\left(D_{\nu}, \xi_{\nu}\right)$ of the form

$$
D_{\nu} f=\nabla_{\nu}\left(v_{f}\right)+\alpha_{\nu} f \mathbf{1}_{\nu}, \quad \mathbf{1}_{\nu}=\mathrm{id}: \xi_{\nu} \rightarrow \xi_{\nu}, \alpha_{\nu} \in i \mathbf{R} .
$$


4. For $\alpha \in \mathbf{C}$ we define an $\alpha$-first-order representation of $(M, \omega)$ to be a firstorder representation $(D, \xi)$ of $(M, \omega)$ such that $D f=\nabla\left(v_{f}\right)+\alpha f 1$, where 1: $\xi \rightarrow \xi$. This $\alpha$ will be referred to as the quantum number of $(D, \xi)$ or, if rank $\xi=k$, as a $k$-quantum number of $(M, \omega)$; it is said to be metrizable, if $(D, \xi)$ is metrizable. Thus, a $k$-quantum number $\alpha$ of $(M, \omega)$ is part of a representing triple $(\xi, \nabla, \alpha i)$ and as such satisfies $K(\nabla)=-\alpha \omega 1$. The geometric definition of Chern classes of $\xi$ yields now

LEMMA 2. If $\alpha$ is a $k$-quantum number of $(M, \omega)$, then the cohomology classes

$$
\left(\begin{array}{l}
k \\
\nu
\end{array}\right) \frac{\alpha^{\nu}}{(2 \pi i)^{\nu}}[\omega]^{\nu} \in H^{2 \nu}(M, \mathbf{C}), \quad \nu=1, \ldots, k,
$$

are integral.

In general, this condition (probably) does not characterize the $k$-quantum numbers of $(M, \omega)$; it does so, however, in the case $k=1$ as explained in $\S 1$, a 1quantum number being a quantum number in the terminology of $\S 1$ (Proposition 1). Let $\tilde{Q}_{m}(M, \omega):=\bigcup_{k \in \mathrm{N}} Q_{m}^{k}(M, \omega)$, where $Q_{m}^{k}(M, \omega)$ is the set of all metrizable $k$-quantum numbers of $(M, \omega)$; from

$$
\left(\xi_{1}, \nabla_{1}, \alpha_{1} 1\right) \cdot\left(\xi_{2}, \nabla_{2}, \alpha_{2} 1\right)=\left(\xi_{1} \otimes \xi_{2}, \nabla_{1} \otimes \nabla_{2},\left(\alpha_{1}+\alpha_{2}\right) 1\right)
$$

and the dual operation $(\xi, \nabla, \alpha \mathbf{1})^{*}=\left(\xi^{*}, \nabla^{*},-\alpha \mathbf{1}\right)$ it follows that $\tilde{Q}_{m}(M, \omega)$ is a subgroup of the additive group $i \mathbf{R} \subset \mathbf{C}$.

5. We compute now the set of (metrizable) first-order representations of $(M, \omega)$. Let $\Sigma(M, \omega)$ denote this set, $\Sigma_{m}(M, \omega)$ the corresponding subset of metrizable triples. For two such triples $T_{1}=\left(\xi_{1}, \nabla_{1}, \psi_{1}\right), T_{2}\left(\xi_{2}, \nabla_{2}, \psi_{2}\right)$, their direct sum:

$$
T_{1}+T_{2}:=\left(\xi_{1} \oplus \xi_{2}, \nabla_{1} \oplus \nabla_{2}, \psi_{1}+\psi_{2}\right)
$$

and tensorproduct:

$$
T_{1} \cdot T_{2}:=\left(\xi_{1} \otimes \xi_{2}, \nabla_{1} \otimes \nabla_{2}, \psi_{1} \otimes 1+1 \otimes \psi_{2}\right)
$$

define on $\Sigma(M, \omega)$ and on $\Sigma_{m}(M, \omega)$ the structure of a commutative half ring. $(\xi, \nabla, 0)$ means $(\xi, \nabla)$ is flat, such that the half ring of flat (complex) vector bundles over $M$-denoted by $\mathcal{F}(M)$, or $\mathcal{F}_{m}(M)$ in the case of metrizable bundles-is a subring of $\Sigma(M, \omega)\left(\Sigma_{m}(M, \omega)\right.$ respectively), and so $\Sigma(M, \omega)\left(\Sigma_{m}(M, \omega)\right.$ resp. $)$ is a left $\mathcal{F}(M)-\left(\mathcal{F}_{m}(M)-\right)$ module.

Proposition 4. Assume the cohomology class $[\omega]=0$ (which implies $M$ noncompact). For each $\alpha \in i \mathbf{R}$, one can choose a representing triple of $(M, \omega) T_{\alpha}=$ $\left(\lambda_{\alpha}, \nabla_{\alpha}, \alpha \mathbf{1}\right)$ with rank $\lambda_{\alpha}=1$, such that the set of isomorphism classes $\left\{\left[T_{\alpha}\right], \alpha \in i \mathbf{R}\right\}$ is a basis of the $\mathcal{F}_{m}(M)$-module $\Sigma_{m}(M, \omega)$.

So, the prequantizations of $\S 1$ generate freely all first-order representations of $(M, \omega)$.

ProOF of Proposition 4. Let $1_{M}$ denote the product bundle $M \times \mathbf{C} \rightarrow M$ with trivial connection $\nabla_{0}$, and $F_{0}:=\left(1_{M}, \nabla_{0}\right)$ the trivial flat bundle. $\left[F_{0}\right]$ is the multiplicative unit of $\Sigma_{m}(M, \omega)$, and $\left[F_{0}\right]=[F]$ for a flat bundle $F=(\lambda, \nabla)$ iff $\lambda$ has a section $s, s(x) \neq 0 \forall x \in M, \nabla s=0$. For $\alpha=i \beta, \beta \in \mathbf{R}$ and $\beta>0$, choose a representing triple $T_{\alpha}=\left(\lambda_{\alpha}, \nabla_{\alpha}, \alpha 1\right)$ according to Proposition 1 , for $\alpha=0$ take 
$T_{0}=\left(1_{M}, \nabla_{0}, 0\right)$ from above, and for $\alpha=i \beta, \beta \in \mathbf{R}$ and $\beta<0$, define $T_{\alpha}$ as the dual of $T-\alpha, T_{\alpha}=\left(\lambda_{-\alpha}^{*}, \nabla_{-\alpha}^{*}, \alpha \mathbf{1}\right)$. Then, $\left[T_{\alpha}\right]\left[T_{-\alpha}\right]=\left[T_{\alpha} T_{-\alpha}\right]=\left[T_{0}\right]=\left[F_{0}\right]$ is the multiplicative unit of $\Sigma_{m}(M, \omega)$. Let now $T$ be a metrizable representing triple of $(M, \omega)$; according to the Corollary, $T$ is a unique sum $T=\sum T_{\nu}$, where $T_{\nu}=\left(\xi_{\nu}, \nabla_{\nu}, \alpha_{\nu} 1\right)$ are metrizable representing triples, $\alpha_{\nu} \in i \mathbf{R}$. For every $\nu$, there is a (up to isomorphism) unique flat bundle $F_{\nu}$ such that $T_{\nu}=F_{\nu} T_{\alpha_{\nu}}$, namely $F_{\nu}=T_{\nu} T_{-\alpha_{\nu}}$.

6. Without the assumption $[\omega]=0$, one has the following information on $\Sigma_{m}(M, \omega)$.

Let $G(R)$ be the Grothendieck ring of a half ring $R$, in particular $G\left(\Sigma_{m}(M, \omega)\right)$ is the representation ring of $P(M, \omega)$-the Poisson algebra of $(M, \omega)$ - and as such contains the subring $G\left(\mathcal{F}_{m}(M)\right)$ of flat bundles. A homomorphism $r: G\left(\mathcal{F}_{m}(M)\right) \rightarrow$ $\mathbf{Z}$ is given by defining $r$ on $\mathcal{F}_{m}(M)$ as the rank of a vector bundle. Then, $P:=$ $r^{-1}(0)$ is a prime ideal of $G\left(\mathcal{F}_{m}(M)\right)$, and for brevity we use the symbols $f_{m}(M)$ and $\sigma_{m}(M, \omega)$ to denote the localizations at $\mathcal{P}$ of $G\left(\mathcal{F}_{m}(M)\right)$ and $G\left(\Sigma_{m}(M, \omega)\right)$ respectively. We localize in this way by the simple reason of making the flat bundles invertible.

Proposition 5. The localized representation ring $\sigma_{m}(M, \omega)$ of $P(M, \omega)$ is a free module over $f_{m}(M)$ with

$$
\operatorname{dim}\left(\sigma_{m}(M, \omega) \mid f_{m}(M)\right)=\tilde{Q}_{m}(M, \omega) .
$$

For the proof one uses Lemma 1 and its Corollary applied to metrizable representing triples of $(M, \omega)$. It states essentially that $\Sigma_{m}(M, \omega)$ is graded by $\tilde{Q}_{m}(M, \omega)$ :

$$
\Sigma_{m}(M, \omega)=\bigoplus_{\alpha} \Sigma_{m}^{\alpha}(M, \omega), \quad \alpha \in \tilde{Q}_{m}(M, \omega),
$$

where $\Sigma_{m}^{\alpha}(M, \omega)$ is the set of isomorphism classes of triples $T_{\alpha}:=(\xi, \nabla, \alpha \mathbf{1})$. Choose for each $\alpha \in \tilde{Q}_{m}(M, \omega)$ such a triple $T_{\alpha}$; using the grading above one then shows that the $T_{\alpha}$ as elements of $\sigma_{m}(M, \omega)$ are a $f_{m}(M)$-basis for this module.

\section{BIBLIOGRAPHY}

1. W. Greub and H. R. Petry, Minimal coupling and complex line bundles, J. Math. Phys. 16 (1975), 1347-1351.

2. G. Karrer, Geometrische Quantisierung, ein Seminarbericht, Mimeographed notes, Math. Inst., Univ. of Zurich, 1979.

3. B. Kostant, Quantization and unitary representation, Lecture Notes in Math., vol. 170, SpringerVerlag, Berlin, 1970, pp. 87-208.

4. R. Narasimhan, Analysis on real and complex manifolds, Masson, Paris; North-Holland, Amsterdam, 1968.

MAThematical Institute, UNIVERSity OF ZURICH, FREIESTRASSE 36, 8032 ZURICH, SWITZERLAND 